\title{
Adressen und Infernetadressen
}

Deutscher Psoriasis Bund e.V.

Seewartenstr. 10

20459 Hamburg, Deutschland

Tel. +49 (0) 40223 399-0

Fax +49 (0) 40223 399-22

info@psoriasis-bund.de

www.psoriasis-bund.de

Bundesarbeitsgemeinschaft Selbsthilfe e.V.

Kirchfeldstr. 149

40215 Düsseldorf, Deutschland

Tel. +49 (0) $21131006-0$

Fax +49(0) 211310 06-48

elisabeth.fischer@bag-selbsthilfe.de

www.bag-selbsthilfe.de

Deutsche Dermatologische Gesellschaft (DDG)

Geschäftsstelle

Robert-Koch-Platz 7

10115 Berlin, Deutschland

Tel. +49 (0) $30246253-0$

Fax +49 (0) 30246 253-29

ddg@derma.de

www.derma.de

Bundesverband der Deutschen

Dermatologen e.V. (BVDD)

Geschäftsstelle

Robert-Koch-Platz 7

10115 Berlin, Deutschland

Tel. +49 (0) 30246 253-53

Fax +49 (0) 30246 253-33

a.bueno@bvdd-berlin.de

www.uptoderm.de

Bundesinstitut für Arzneimittel und

Medizinprodukte (BfArM)

Kurt-Georg-Kiesinger-Allee 3

53175 Bonn, Deutschland

Tel. +49 (0) 228 207-30

Fax +49 (0) 228 207-5207

pressestelle@bfarm.de

www.bfarm.de

Verband Forschender

Arzneimittelhersteller e.V.

Hausvogteiplatz 13

10117 Berlin, Deutschland
Tel. +49 (0) 30206 04-0

Fax +49(0) 30206 04-222

info@vfa.de

www.vfa.de

Psoriasis Selbsthilfe

Arbeitsgemeinschaft e.V. (PSOAG)

Schmitzweg 64

13437 Berlin, Deutschland

Tel./Fax +49 (0) 3061283090

psoag@gmx.de

www.psoriasis-selbsthilfe.org

Schweizerische Psoriasis-Vitiligo

Gesellschaft (SPVG)

Gryphenhübeliweg 38

Postfach 345

3000 Bern 6, Schweiz

Tel. + 41 (0) 31359 90-18

Fax + $41(0) 31359$ 90-90

info@spvg.ch

www.spvg.ch

PSO-Austria

Selbsthilfeverein

Obere Augartenstr. 26-28/1.18

1020 Wien, Österreich

Tel. +43 (0) 66473111991

office@pso-austria.org

www.pso-austria.org

Letzebuerger

Psoriasisbond a.s.b.l.

25, rue Léon Jouhaux

4155 Esch/Alzette, Luxemburg

Tel. +3 52 (0) 550478 oder +352(0) 0779

International Federation

of Psoriasis Associations

Box 5173

12118 Johanneshov, Schweden

Tel. +46 (0) 855610914

Fax +46 (0) 855610919

annika.rastas@pso.se

www.ifpa-pso.org

National Psoriasis Foundation

(US-amerikanische Selbsthilfe)

www.psoriasis.org 